Revue européenne des sciences sociales

European Journal of Social Sciences

54-1 | 2016

Les usages sociaux des sciences du vivant

\title{
Jean-François LAÉ, Dans l'œil du gardien
}

\section{Lionel Francou}

\section{(2) OpenEdition}

\section{Journals}

Édition électronique

URL : http://journals.openedition.org/ress/3174

DOI : $10.4000 /$ ress. 3174

ISBN : $1663-4446$

ISSN : 1663-4446

Éditeur

Librairie Droz

Édition imprimée

Date de publication : 15 mai 2016

Pagination : 280-282

ISSN : 0048-8046

Référence électronique

Lionel Francou, « Jean-François LAÉ, Dans l'œil du gardien », Revue européenne des sciences sociales [En ligne], 54-1 | 2016, mis en ligne le 15 mai 2016, consulté le 22 septembre 2020. URL : http:// journals.openedition.org/ress/3174; DOI : https://doi.org/10.4000/ress.3174

Ce document a été généré automatiquement le 22 septembre 2020

(C) Librairie Droz 


\title{
Jean-François LAÉ, Dans l'œil du gardien
}

\author{
Lionel Francou
}

\section{RÉFÉRENCE}

Jean-François LAÉ, 2015, Dans l'œil du gardien, Paris, Éditions du Seuil, « Raconter la vie $», 102$ p.

1 La vulgarisation scientifique est, il faut bien le reconnaître, le parent pauvre des sciences (sociales). Proportionnellement aux autres tâches qui occupent les (enseignants-)chercheurs, peu de moyens sont déployés pour les encourager à diffuser le produit de leurs recherches au plus grand nombre ou, tout simplement, pour leur rendre une telle démarche possible. Les sciences sociales doivent en plus composer avec le fait que leurs objets de recherche sont, le plus souvent, directement liés à l'expérience que peuvent en faire eux-mêmes les acteurs qu'ils observent. Dès lors, comment porter un discours structuré, nuancé et clair (en évitant d'être trop formel et abscons) dans le débat public, tout en se rendant audible face aux essayistes flamboyants, aux témoignages dramatiques ou aux simplifications éhontées - qu'elles soient ou non faites de bonne foi ? La collection «Raconter la vie ", dirigée par les historiens Pierre Rosanvallon et Pauline Peretz, se donne pour objectif de rassembler de courts textes, de scientifiques et d'écrivains, notamment, à même de produire « le roman vrai de la société française ». Elle a le mérite de proposer un format qui permet de rendre accessible et agréable à lire pour un large public le fruit d'enquêtes proposant des pistes d'analyse en prise avec la réalité sociale et, plus spécifiquement, avec la société française considérée sous ses multiples facettes.

2 Dans le présent ouvrage, Jean-François Laé, professeur de sociologie à l'Université Paris 8 Vincennes-Saint-Denis, auteur de Les Nuits de la main courante (Stock, 2008), dans lequel il s'intéressait aux cahiers utilisés dans différents lieux, dont une cité HLM, s'attaque désormais à une problématique au croisement des questions de « rénovation 
urbaine » et de « médiation sociale », à savoir le métier de gardien en cité HLM. Pour se familiariser avec cette profession et avec le quotidien de ceux qui l'exercent, il a mené quelques observations dans une cité du «93», en Seine-Saint-Denis, et s'est entretenu avec les premiers concernés et leurs collègues directs. L'ouvrage est d'ailleurs une sorte de recueil de descriptions de ce qu'a pu voir l'auteur, de ses ressentis et impressions, ainsi que d'extraits de discussions issus de l'enquête qu'il a menée fin 2013-début 2014. Quelques enquêtés - furent-ils les seuls? - sont particulièrement mobilisés dans le texte, que ce soit pour décrire leurs actions ou rapporter leurs paroles : trois gardiens, un formateur de gardiens et une agente d'accueil. Au vu de la structure inhabituelle de l'ouvrage (dix très courts chapitres), nous chercherons surtout à mettre en avant quelques enjeux qui y sont présentés comme centraux.

Un premier point développé transversalement dans cet ouvrage relève de la description, par l'auteur, des évolutions importantes que le métier de gardien a connues ces dernières années. L'ancienne figure du concierge (acariâtre ou bonhomme), retiré dans une loge miteuse, a cédé sa place à un acteur de terrain professionnalisé, revalorisé et présenté comme central dans la vie de la cité. Il n'est plus en charge de l'ensemble des problèmes qui y surviennent, mais en partage la gestion avec un agent d'accueil et un technicien. Il fait sa tournée, constate les incidents, nettoie les espaces communs, mais a aussi un rôle relationnel important. Recrutés par dizaines de milliers à travers la France, ils ont un niveau d'études plus élevé qu'auparavant $(\mathrm{bac}+2)$. Si leur salaire est assez faible, le fait de pouvoir se loger en famille, avec de meilleures conditions de travail que par le passé, a pour conséquence que « le métier est de nouveau convoité » (p. 13).

L'auteur porte ensuite un regard assez critique sur les programmes de rénovation urbaine qui transforment (ou sont censés transformer ?) la cité, à l'image du chantier en cours depuis déjà près de dix ans et engagé après les émeutes urbaines de 2005. Les tours et barres HLM sont progressivement remplacées par de plus petits immeubles par la magie de la "résidentialisation". Les pouvoirs publics cherchent à refaçonner le quartier pour en améliorer l'image, en démolissant et en reconstruisant différemment, mais aussi en communiquant via des panneaux et des affiches, ou par l'usage et la promotion d'un vocabulaire connoté plus positivement pour parler du quartier, des logements et des personnes. Comme le constate l'auteur, «la rénovation urbaine est en mode chasse-neige» (p.15) et, tandis que l'environnement en bénéficie, les plus pauvres doivent s'en aller au profit de nouvelles populations, à même de payer des loyers plus élevés et de remplir des quotas censés garantir une plus grande «mixité sociale ». Ces modifications cosmétiques se font visibles, tandis que sont invisibilisées les populations les plus fragiles et l'accumulation des maux dont elles souffrent.

Enfin, l'auteur souligne également le fait que le rôle principal que doit jouer le gardien est d'ordre relationnel. Il est en contact avec les habitants au quotidien, les connaît - de vue ou de façon plus personnelle - et est connu d'eux. Son rapport à la cité est familier et il se trouve constamment en contact avec la population. D'après une circulaire portant sur son métier, le gardien doit à la fois assurer une mission de gardiennage et de surveillance, ainsi que se "rapprocher plus encore des habitants» (p.60). Pour l'auteur, «le contrôle des conduites a tout simplement changé de nom» (p. 64). Le gardien, devenu «médiateur», doit "amortir les chocs» (p.64), "faire la paix, produire du respect » (p. 61), prévenir et guérir les conflits (tous les conflits ?), filer un coup de main, faire en sorte que tout se passe bien, en somme. Il mène au quotidien 
« une activité de ralliement » (p. 30), ce qui peut l'amener à « faire des petits gestes en plus» (p. 75), pour créer et consolider une relation de confiance avec les habitants. «Réparateurs de la vie collective »(p. 75), les gardiens se retrouvent dans des positions pour le moins ambivalentes lorsqu'ils choisissent de fermer les yeux sur certains désordres pour ne pas envenimer les choses ou quand leur cœur balance entre le respect de l'ordre et la compassion pour des situations humaines difficiles. Pour l'auteur, ils sont les acteurs d'« un mode de gouvernement par le bas» (p. 90), chargés d'aider les gens mais aussi (et surtout ?) de les responsabiliser et de les garder à l'œil. Dans une telle fonction, aux contours assez flous et potentiellement contradictoires, il est difficile de poser des limites : où doivent-ils s'arrêter? Et, finalement, quels sont véritablement leur impact et leur marge de manœuvre?

6 En définitive, ce petit livre très accessible contribue à une sociologie qui cherche à faire entendre ses analyses au-delà du cercle assez restreint de ses lecteurs habituels. Ce résultat n'est possible qu'en faisant fi des règles habituelles qui encadrent la présentation des résultats d'une recherche scientifique. Si cela ne gênera probablement pas la majorité des lecteurs, il faut néanmoins noter que ce texte ne donne pas plus de détails sur la méthodologie à laquelle a recouru l'auteur ou sur le contexte dans lequel s'est inscrite cette recherche. Si l'on devine certaines inclinaisons au fil du texte, le format empêche aussi Jean-François Laé de développer plus en profondeur ses analyses. Bien qu'il attire l'attention du lecteur sur une série de problèmes sociaux, il ne le fait qu'assez brièvement, sans être véritablement en mesure de préciser sa pensée et les implications potentielles de celle-ci. L'exercice de vulgarisation scientifique n'est pas aisé, et il faut féliciter l'auteur pour avoir relevé ce défi avec succès, d'autant plus sur un thème d'actualité.

\section{AUTEURS}

\section{LIONEL FRANCOU}

Université catholique de Louvain, CriDIS 